\title{
Early Osteoarthritis and Double-Layered Patella in a Patient With Multiple Epiphyseal Dysplasia
}

\author{
Selçuk SAYILIR, ${ }^{1}$ Timur EKİZ ${ }^{2}$ \\ ${ }^{1}$ Department of Physical Medicine and Rehabilitation, Muğla Sıtkı Koçman University Medical School, Muğla, Turkey \\ ${ }^{2}$ Department of Physical Medicine and Rehabilitation, Dermancan Medical Center, Adana, Turkey
}

\begin{abstract}
Multiple epiphyseal dysplasia is a relatively rare skeletal dysplasia characterized with early osteoarthritis resulting in joint dysfunction and short stature in childhood. In this article, we presented a 42-year-old patient of multiple epiphyseal dysplasia who was newly diagnosed, had relatively normal height, osteoarthritis, and double-layered patella. The patient was diagnosed in adulthood in contrast to the literature because the clinical scenario was apparent in the late periods.

Keywords: Double-layered patella; osteoarthritis; skeletal dysplasia.
\end{abstract}

Skeletal dysplasia refers to a diverse and heterogeneous group affecting skeletal maturation, development, and stature. Although rare with a prevalence of $2-5 / 10,000$ at birth, more than 400 different types of skeletal disorders have been previously described. ${ }^{1-3}$ They are classified/ named according to radiologic findings, affected parts of the skeleton (e.g. multiple epiphyseal dysplasia [MED], spondylometaphyseal dysplasia), eponyms (e.g. Stickler dysplasia, Ellis-van Creveld dysplasia,) or with some special terms (e.g. diastrophic dysplasia, opsismodysplasia). ${ }^{3,4}$

Multiple epiphyseal dysplasia is a relatively rare skeletal dysplasia characterized with early osteoarthritis (OA) resulting in joint dysfunction, particularly in knee and hip joints, and short stature in childhood. Since it can present mildly, proper diagnosis can be difficult. ${ }^{5}$ It is a genetically heterogeneous disease and its exact mechanism is not clearly known. However, some causative genes resulting in maturational delay in the epiphysis of the bone have been previously reported. ${ }^{6}$ Conservative treatment methods are applied as the genetic mechanisms play a role in the pathophysiology. Accordingly, in this article, we presented a case of MED with widespread OA whereby a late diagnosis of MED was highlighted.

\section{CASE REPORT}

A 42-year-old male patient was examined due to gait difficulty and pain in hip and knee joints bilaterally and low back. He declared that he could walk independently, yet he had some difficulties in walking on undulating surfaces or climbing up stairs. Medical and family history was noncontributory. He was $168 \mathrm{~cm}$ tall and weighed $78 \mathrm{~kg}$. Physical examination revealed severe restriction of range of motion in hip joints, crepitation in knee joints, hyperextension position of the first metacarpophalangeal joint, and deviation of the thumbs. Adam's test for scoliosis was positive and lumbar motions were limited as well. Minimal joint effusion was detected in knee 

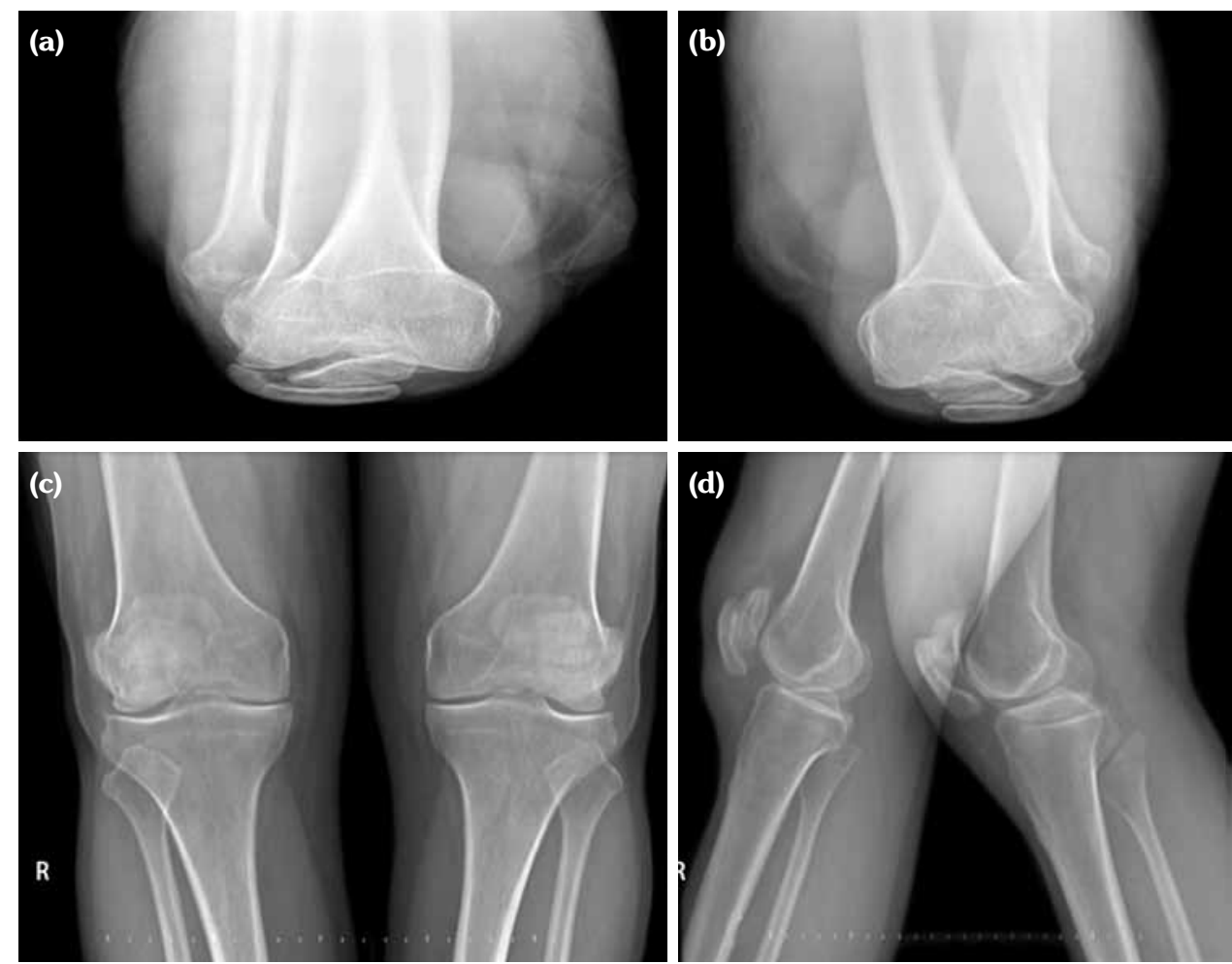

Figure 1. Knee radiographs show a complete double-layered patella in right (a) and left (b) side. Anteroposterior (c) and lateral (d) knee radiographs demonstrate uniform narrowing of joint space, subchondral sclerosis, and degenerative changes in joints.

joints as well. Knee radiographies showed doublelayered patella (DLP), generalized degenerative changes, scoliosis, and circumferential radiopaque lesion around the right hip joint (calcification of the iliopsoas bursa) (Figures 1 and 2). Magnetic resonance imaging designated the association
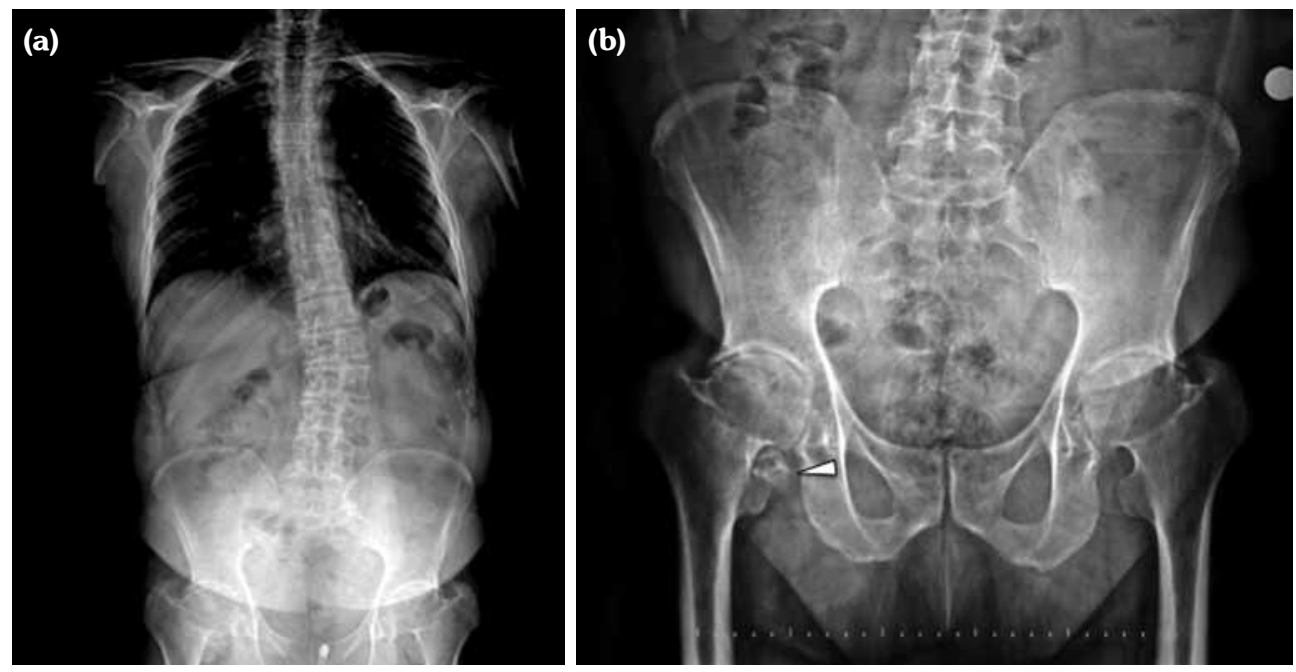

Figure 2. Spinal radiograph demonstrates scoliosis in thoracolumbar region (a). Pelvis radiograph illustrates degenerative changes in acetabular and femoral surfaces, osteophytes, narrowed joint spaces, short femoral necks and well-defined calcification (arrowhead) near right trochanter minor (b). 


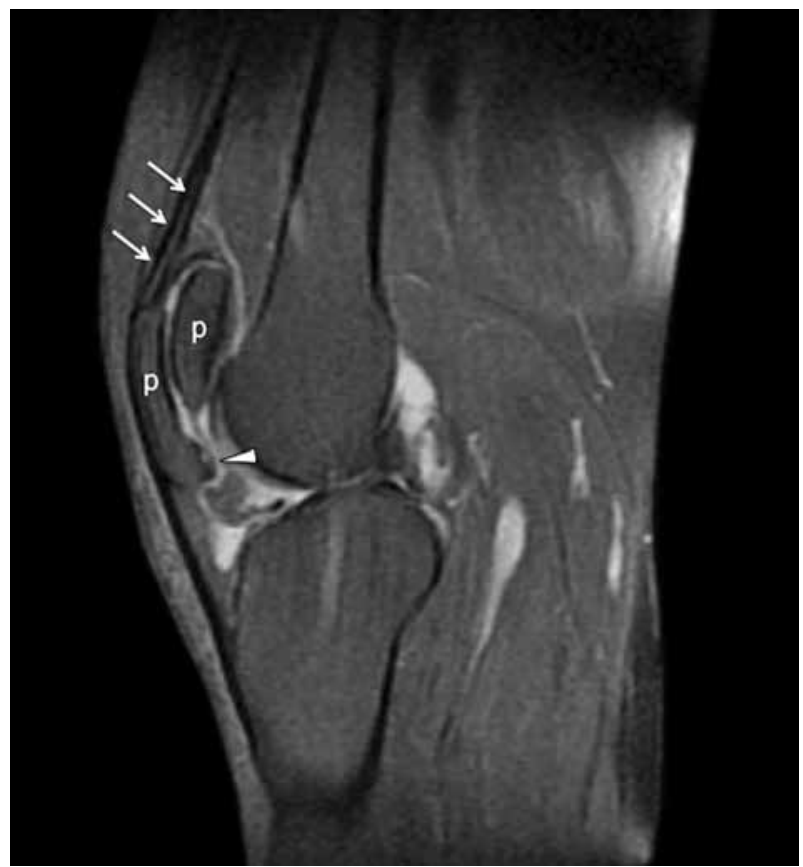

Figure 3. Magnetic resonance imaging (sagittal, $\mathrm{T}_{2}$-view) shows joint effusion and degenerative changes (arrowhead). Distal part of double-layered patella ( $p$ ) is associated with quadriceps tendon (thin arrows), whereas proximal part is not.

between the quadriceps tendon and patella (Figure 3). Laboratory investigations including erythrocyte sedimentation rate and C-reactive protein, calcium, phosphate, and alkaline phosphatase levels were all within normal limits. Overall, the patient was diagnosed with secondary OA due to MED and referred to another center for further genetic analysis. A written informed consent was obtained from the patient.

\section{DISCUSSION}

Multiple epiphyseal dysplasia is a rare cause of secondary $\mathrm{OA}$ and widespread pain. Its signs/ symptoms are mechanical joint pain and deviation from the normal curve by the age of five or six. Therefore, most patients are diagnosed with MED in childhood. Kim et al. ${ }^{7}$ reported 40 patients with MED with a mean age of 9.6 years. In contrast, our patient was diagnosed in adulthood. Although MED usually results in short stature, our patient had a relatively normal stature -as in mild forms of MED- and this could be confusing for proper diagnosis. MED is usually inherited in an autosomal dominant manner. ${ }^{1,3}$ However, our patient had no remarkable family history. We could not administer a genetic analysis in our institution and referred the patient to another center. Yet our patient's condition may have been related to autosomal recessive or other subgroups of MED, being quite rare.

Our patient with clunking in the knees had DLP bilaterally, which was reported previously in the literature. ${ }^{8}$ DLP is a type of bipartite patella and caused by a septum dividing the patella into segments. It can be seen completely or partially. In general, DLP is associated with MED; however, it is reported in nondysplastic subjects as well. ${ }^{9}$ Our patient had the total form of DLP. Magnetic resonance imaging showed that the distal part of the DLP was responsible for the extensor mechanism of the knees. This condition is important because the functional part could be indicative for a possible surgical procedure or functionality of the knee movements.

Accordingly, by presenting our unusual case, we would like to highlight that MED may be diagnosed in adulthood in case of mild forms. Therefore, MED should be considered for the differential diagnosis of early/secondary OA.

\section{Declaration of conflicting interests}

The authors declared no conflicts of interest with respect to the authorship and/or publication of this article.

\section{Funding}

The authors received no financial support for the research and/or authorship of this article.

\section{REFERENCES}

1. Rasmussen SA, Bieber FR, Benacerraf BR, Lachman RS, Rimoin DL, Holmes LB. Epidemiology of osteochondrodysplasias: changing trends due to advances in prenatal diagnosis. Am J Med Genet 1996;61:49-58.

2. Bonafe L, Cormier-Daire V, Hall C, Lachman R, Mortier G, Mundlos S, et al. Nosology and classification of genetic skeletal disorders: 2015 revision. Am J Med Genet A 2015;167A:2869-92.

3. Briggs MD, Chapman KL. Pseudoachondroplasia and multiple epiphyseal dysplasia: mutation review, molecular interactions, and genotype to phenotype correlations. Hum Mutat 2002;19:465-78. 
4. van Mourik JB, Hamel BC, Mariman EC. A large family with multiple epiphyseal dysplasia linked to COL9A2 gene. Am J Med Genet 1998;77:234-40.

5. Mäkitie O, Mortier GR, Czarny-Ratajczak M, Wright MJ, Suri M, Rogala P, et al. Clinical and radiographic findings in multiple epiphyseal dysplasia caused by MATN3 mutations: description of 12 patients. Am J Med Genet A 2004;125A:278-84.

6. Unger S, Bonafé L, Superti-Furga A. Multiple epiphyseal dysplasia: clinical and radiographic features, differential diagnosis and molecular basis.
Best Pract Res Clin Rheumatol 2008;22:19-32.

7. Kim SJ, Ramanathan AK, Jeon YS, Song HR. The fate of hips that are conservatively treated in multiple epiphyseal dysplasia. J Pediatr Orthop B 2016 Jul 8. [Epub ahead of print]

8. Sheffield EG. Double-layered patella in multiple epiphyseal dysplasia: a valuable clue in the diagnosis. J Pediatr Orthop 1998;18:123-8.

9. García-Mata S, Hidalgo-Ovejero A. Partial doublelayered patella in a nondysplasic adolescent. J Pediatr Orthop B 2016;25:561-3. 